CARDIOVASCULAR MEDICINE

\title{
Long term cardioprotective action of trimetazidine and potential effect on the inflammatory process in patients with ischaemic dilated cardiomyopathy
}

\author{
P Di Napoli, A A Taccardi, A Barsotti
}

Heart 2005;91:161-165. doi: 10.1136/hrt.2003.031310

\begin{abstract}
See end of article for authors' affiliations

Correspondence to: Dr Pericle Di Napoli, Dipartimento di Cardiologia-UTIC, Casa di Cura Villa Pini d'Abruzzo, Via dei Frentani 228, 66100 Chieti, Italy; dinapoli@unich.it
\end{abstract}

Accepted 2 June 2004

\begin{abstract}
Objective: To investigate the long term effects of trimetazidine in patients with dilated ischaemic cardiomyopathy. The effects of trimetazidine on left ventricular function as well as its tolerability profile and potential anti-inflammatory effects were studied.

Design: 61 patients were randomly assigned either to receive trimetazidine (20 $\mathrm{mg}$ thrice daily) in addition to their conventional treatment or to continue their usual drug treatment for 18 months. Patients were evaluated at baseline and at 6, 12, and 18 months with a clinical examination, echocardiography, and biochemical analysis (C reactive protein).

Results: Trimetazidine added to the usual treatment significantly improved the patients' functional status (assessed by New York Heart Association functional class). The functional improvement of trimetazidine treated patients was associated with a significant increase in left ventricular ejection fraction $(3016) \%, 32$ $(8) \%, 38(7) \%$, and $37(6) \%$ v $31(8) \%, 30(7) \%, 28(6) \%$, and $26(9) \%$ in control patients at baseline and at 6,12 , and 18 months, respectively) and with a significant effect on ventricular remodelling. $C$ reactive protein plasma concentrations remained stable throughout the study in patients receiving trimetazidine (2.5 (1.0), $2.7(2.0), 2.7(3.0)$, and $3.0(2.0) \mathrm{mg} / \mathrm{l}$ at baseline and at 6, 12, and 18 months, respectively) but increased significantly in the control group (2.4 (1.0), 3.4 (1.2), 6.0 (4.0), and 7.0 (5.0) mg/l, respectively). No significant adverse event or changes in clinical or biochemical parameters were detected. Conclusion: Treatment with trimetazidine added to the usual treatment for up to 18 months was well tolerated and induced a functional improvement in patients with dilated cardiomyopathy. Trimetazidine treatment was associated with a significant improvement of left ventricular function and the remodelling process. Results also suggest that the inflammatory response was limited in patients treated with trimetazidine.
\end{abstract}

T imetazidine (1-[2,3,4-trimethoxybenzyl] piperazine dihydrochloride) is the first of a novel antianginal drug class, the selective inhibitors of mitochondrial long chain 3-ketoacyl coenzyme A thiolase. Trimetazidine is widely used for the prophylactic treatment of episodes of angina pectoris at recommended daily doses ranging from 40-60 mg.

The anti-ischaemic properties of trimetazidine are unrelated to changes in the myocardial oxygen supply to demand ratio, as heart rate, blood pressure, and pressure-rate product do not change significantly either at rest or during exercise. ${ }^{2}$ Trimetazidine acts by reducing fatty acid oxidation and by stimulating glucose oxidation. ${ }^{3}{ }^{4}$ Subsequently the coupling of glycolysis with glucose oxidation is improved, ATP production is increased, and the deleterious consequences of acidosis and intracellular calcium ion overload that characterise the ischaemic or hypoxic cell are limited.

The antianginal properties of trimetazidine were previously shown in acute and chronic experimental conditions in which the cardioprotective effects were related to the positive effects on energy metabolism, hydroionic balance, coronary microcirculation, and oxidative stress. ${ }^{56}$ Few data are available regarding the efficacy of trimetazidine in patients with dilated cardiomyopathy. ${ }^{78}$ However, long term data ( $>$ l year) on the safety and usefulness of this treatment in such patients have not been fully explored; nor has its effects on the inflammatory process, an independent negative prognostic factor in patients with coronary artery disease. ${ }^{9-11}$

The aim of this randomised study in patients with ischaemic dilated cardiomyopathy already receiving conventional treatment was to investigate: firstly, the long term effects of trimetazidine (20 mg thrice daily); secondly, its tolerability profile; and thirdly, its effects on the inflammatory process. Patients were followed up for up to 18 months after their inclusion in the study. Efficacy was assessed by evaluating the patients' functional status (New York Heart Association (NYHA) functional class) and left ventricular (LV) function (echographic changes in the LV ejection fraction (LVEF) and volumes). The effect of trimetazidine on the inflammatory process was evaluated by determining $\mathrm{C}$ reactive protein plasma concentrations.

\section{METHODS}

\section{General methods}

This randomised pilot study was conducted at a single site (Villa Pini, Centre for Study and Treatment of Congestive Heart Failure, Chieti, Italy). The number of patients was chosen on the basis of previous studies with trimetazidine performed with a similar design. ${ }^{7}$ The study was conducted in an open label design for patient convenience. Echocardiograms (for primary outcome) were recorded and rated blindly by two independent investigators.

The research protocol was approved by the local appointed ethics committee. All patients provided written informed consent before participation. The enrolment period took place from January 1997 through June 1997 and the last patient visit occurred in January 1999.

Abbreviations: $A C E$, angiotensin converting enzyme; $L V$, left ventricular; LVEF, left ventricular ejection fraction; NYHA, New York Heart Association 


\section{Inclusion criteria}

Patients were included if they had previously had a myocardial infarction and a depressed LVEF $(<40 \%)$ as shown by echography. Patients had undergone a coronarography and they all presented with coronary lesions that were unsuitable for mechanical (that is, percutaneous transluminal coronary angioplasty) or surgical revascularisation.

Patients were excluded if they had had a recent $(<3$ months) acute myocardial infarction or had acute heart failure or cardiac decompensation, renal insufficiency (creatinine $>221 \mu \mathrm{mol} / \mathrm{l}$ ), or chronic pulmonary or systemic disease.

\section{Study treatments}

Patients were randomly divided into two homogeneous groups (ratio 1:1): the trimetazidine group, who received trimetazidine $60 \mathrm{mg}$ ( $20 \mathrm{mg}$ thrice daily) in addition to their conventional treatment; and the control group, who continued their usual drug treatment. The randomisation was provided by individual sealed envelopes prepared in advance at the investigation site.

Throughout the entire study and when necessary the investigator was allowed to modify the dose of conventional treatment (that is, diuretics or angiotensin converting enzyme (ACE) inhibitors) and to lower the dose of trimetazidine or to discontinue or stop the drug if significant adverse effects occurred.

\section{Investigations}

After the randomisation process, patients attended a total of four visits (baseline and 6, 12, and 18 month visits). At each visit they underwent clinical inquiry for adverse events, clinical examination, vital signs, and assessment of their functional status (NYHA class). A 12 lead standard ECG and chest radiograph were also recorded. A cardiac echogram (Acuson, Sequoia Ultrasound System, Mountain View, California, USA) was recorded and analysed blindly by two expert cardiologists (interobserver variability $<2 \%$ ) who determined LV volumes and LVEF by Simpson's method. Standard blood tests were performed including ultrasensitive $\mathrm{C}$ reactive protein determination. ${ }^{9}$

\section{Analysis}

The primary outcome was the change in the echographic parameters (LVEF and LV end diastolic and end systolic volumes) up to the 18 month visit. Secondary outcomes were the evolution of the patients' functional status during the 18 month period and the change in plasma $C$ reactive protein concentrations as a marker of systemic inflammation.

Statistical analysis was performed with Primer (Windows) 4.02i statistical software. Data were analysed according to the randomised assignments regardless of the patient's subsequent medication status. Comparability of both groups at baseline was confirmed by Student's $t$ test for independent series. Parametric variables were compared by a two way analysis of variance (time and treatment) with repeated measurement for the time factor. Qualitative variables were analysed by Fisher's test with Yates's correction for small samples, when required. The level of significance was set at $5 \%(\mathrm{p}<0.05)$.

\section{RESULTS}

\section{Patient population}

Eighty eight patients with ischaemic dilated cardiomyopathy were enrolled. Of these patients, 27 were subsequently excluded because of a poor acoustic window (10), poor motivation (11), or insufficient compliance with the treatment design (6). Thus, 61 patients were enrolled and randomly assigned in the study (trimetazidine group, $\mathrm{n}=30$; control group, $\mathrm{n}=31$ ). Both groups were comparable at baseline with regard to their clinical characteristics, blood testing, echographic evaluation, and distribution of their concomitant treatment. Table 1 describes the study population at baseline.

All patients $(n=61)$ attended the six month visit, 57 patients (trimetazidine group, 28; control group, 29) attended the 12 month visit, and 54 patients (trimetazidine group, 26; control group, 28) completed the last 18 month visit. Seven patients died during the evaluation period. The cause of death was not related to the study treatment or to the study procedures: in the trimetazidine group, three patients died of heart failure and one patient of lung cancer; in the control group, three patients died of irreversible heart failure.

Only a few treatment adjustments were noted during the evaluation period. In the trimetazidine group, two patients increased their dose of diuretics to optimise the clinical compensation. Trimetazidine treatment did not lead to any modification or discontinuation during this period. In the control group, the diuretic treatment was increased for four patients, ACE inhibitor doses were increased for three patients, and digoxin was introduced for two patients.

\begin{tabular}{|c|c|c|}
\hline & $\begin{array}{l}\text { Trimetazidine treated } \\
\text { group }(n=30)\end{array}$ & $\begin{array}{l}\text { Control group } \\
(n=31)\end{array}$ \\
\hline Age (years) & $67(5.5)$ & $69(7)$ \\
\hline Men/women & $17 / 13$ & $18 / 13$ \\
\hline \multicolumn{3}{|l|}{ Drug treatment } \\
\hline Diuretics & $30(100 \%)$ & $31(100 \%)$ \\
\hline ACE inhibitors or angiotensin II antagonists & $27(90 \%)$ & $28(90.3 \%)$ \\
\hline Digoxin & $8(26.7 \%)$ & $7(22.6 \%)$ \\
\hline Nitrates & $6(20 \%)$ & $8(25.8 \%)$ \\
\hline$\beta$ Blockers & $15(50 \%)$ & $18(58.1 \%)$ \\
\hline Calcium antagonists & $6(20 \%)$ & $6(19.4 \%)$ \\
\hline Aspirin & $22(73.3 \%)$ & $20(64.5 \%)$ \\
\hline \multirow{2}{*}{\multicolumn{3}{|c|}{$\begin{array}{l}\text { Echocardiography } \\
\text { left ventricular volume }\end{array}$}} \\
\hline Left ventricular volume & & \\
\hline End systolic $\left(\mathrm{ml} / \mathrm{m}^{2}\right)$ & $91(18)$ & $88(15)$ \\
\hline End diastolic $\left(\mathrm{ml} / \mathrm{m}^{2}\right)$ & $133(30)$ & $127(22)$ \\
\hline Ejection fraction (\%) & $30(6)$ & $31(8)$ \\
\hline Mitral regurgitation (light to moderate) & $17(56.7 \%)$ & $16(51.6 \%)$ \\
\hline
\end{tabular}

Parametric data are expressed as mean (SD) and non-parametric data as number of patients (\%). $\mathrm{ACE}$, angiotensin converting enzyme. 
Table 2 Evolution of the patients' functional status by New York Heart Association functional class

\begin{tabular}{|c|c|c|c|c|c|c|c|c|c|c|}
\hline \multirow[b]{2}{*}{ Time point } & \multicolumn{5}{|c|}{ Trimetazidine group } & \multicolumn{5}{|c|}{ Control group } \\
\hline & I & II & III & IV & Total & 1 & II & III & IV & Total \\
\hline Baseline & 0 & 11 & 17 & 2 & 30 & 0 & 10 & 18 & 3 & 31 \\
\hline 6 months & 1 & 11 & 16 & 2 & 30 & 0 & 10 & 17 & 4 & 31 \\
\hline 12 months & 4 & 20 & 4 & 0 & 28 & 0 & 2 & 23 & 4 & 29 \\
\hline 18 month & 4 & 21 & 1 & 0 & 26 & 0 & 1 & 24 & 3 & 28 \\
\hline
\end{tabular}

\section{Functional status}

Table 2 details the patients' functional status during the evaluation period. At the 12 and 18 month visits, a significant functional improvement was noted in most patients receiving trimetazidine added to their usual treatment, as shown by their distribution in the NYHA functional class (mean NYHA class after 12 months $2.03(0.63)$ in the trimetazidine group, $3.06(0.45)$ in the control group, $\mathrm{p}<0.001$; after 18 months $1.88(0.43)$ in the trimetazidine group, $3.03(0.42)$ in the control group, $\mathrm{p}<0.001$ ).

Compared with baseline, 20 patients from this group gained one NYHA class, mostly after 12 months of treatment with trimetazidine; four patients had no change and only one patient experienced a functional worsening from grade II to grade III. In contrast, a functional improvement was observed in only one patient from the control group (from grade III to grade II), whereas NYHA status remained unchanged in 15 patients and deteriorated in 12 patients compared with baseline.

\section{LV function}

Figures 1 and 2 show the changes in echocardiographic parameters. In patients from the trimetazidine group, an increase of LVEF was noted, starting at six months and maintained after 12 and 18 months of treatment with trimetazidine (mean (SD) $30(6) \%, 32(8) \%, 38(7) \%$, and $37(6) \%$ at baseline and at 6, 12, and 18 months, respectively). In contrast, LVEF deteriorated in patients from the control group during the same period (mean (SD) 31 $(8) \%, 30(7) \%, 28(6) \%$, and $26(9) \%$ at baseline and at 6,12 , and 18 months, respectively). The increase in LVEF in patients receiving trimetazidine added to their usual treatment was significant at 12 months $(\mathrm{p}<0.001)$ and 18 months $(p<0.001)$ compared with patients taking their usual medications.

The increase of LVEF in the trimetazidine group was associated with a significant reduction of the LV volumes. In

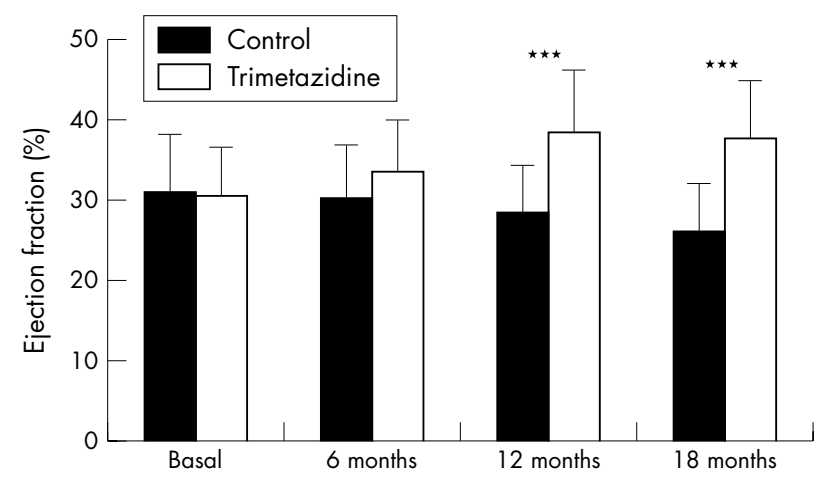

Figure 1 Changes in the ejection fraction in patients treated with (trimetazidine group) or without (control group) trimetazidine in addition to their usual medication. Data are mean (SD). ${ }^{* * *} p<0.001 v$ control.

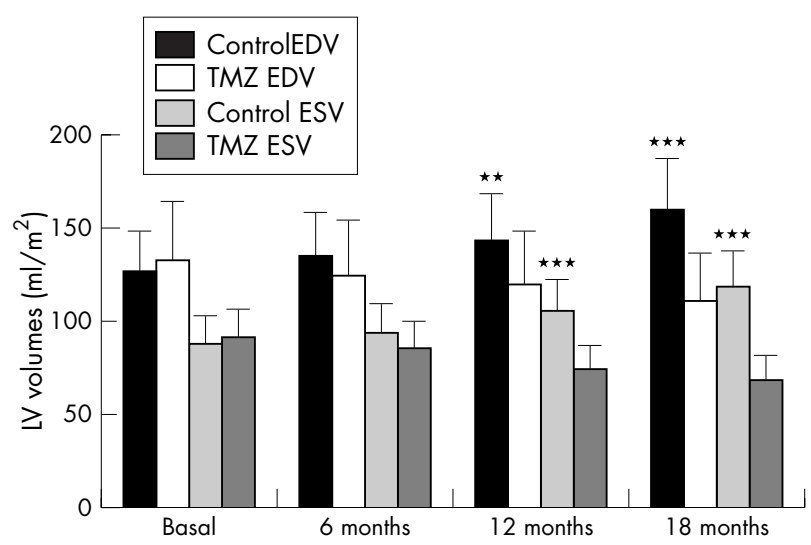

Figure 2 Changes in the left ventricular (LV) volumes. Data are mean (SD). ${ }^{* *} p<0.01 ;{ }^{* * *} p<0.001 v$ trimetazidine. EDV, end diastolic volume; ESV, end systolic volume; TMZ, trimetazidine.

contrast, the LV volumes progressively increased in the control group from baseline up to 18 months. The differences were significant at 12 months $(\mathrm{p}<0.01, \mathrm{p}<0.001$, end diastolic and end systolic volume, respectively) and 18 months (both volumes: $\mathrm{p}<0.001$ ).

\section{C reactive protein plasma concentrations}

As fig 3 shows, C reactive protein plasma concentrations remained stable throughout the study in patients from the trimetazidine group (2.5 (1.0) mg/l, 2.7 (2.0) mg/l, 2.7 (3.0) $\mathrm{mg} / \mathrm{l}$, and $3.0(2.0) \mathrm{mg} / \mathrm{l}$ at baseline and at 6,12 , and 18 months, respectively). In comparison, $\mathrm{C}$ reactive protein concentrations increased significantly in the control group $(2.4 \quad(1.0) \mathrm{mg} / \mathrm{l}, \quad 3.4 \quad(1.2) \mathrm{mg} / \mathrm{l}, \quad 6.0 \quad(4.0) \mathrm{mg} / \mathrm{l}$, and 7.0 (5.0) $\mathrm{mg} / \mathrm{l}$ at baseline and at 6, 12, and 18 months, respectively). The difference between the groups was significant at the 12 month and 18 month assessments $(\mathrm{p}<0.01)$.

\section{Safety}

Vital signs remained unchanged throughout the evaluation period. No significant changes in blood determination of renal and hepatic function or serum electrolytes were detected. Only one patient reported transient headache related to trimetazidine treatment according to the investigating physician but did not have to discontinue treatment. No other significant adverse event was notified. In conclusion, patients with ischaemic dilated cardiomyopathy tolerated trimetazidine well during up to 18 months of treatment.

\section{DISCUSSION}

The present study indicated that trimetazidine added to usual treatment improved the functional status of patients with ischaemic dilated cardiomyopathy, as shown by their distribution in the NYHA class after 18 months of treatment. 


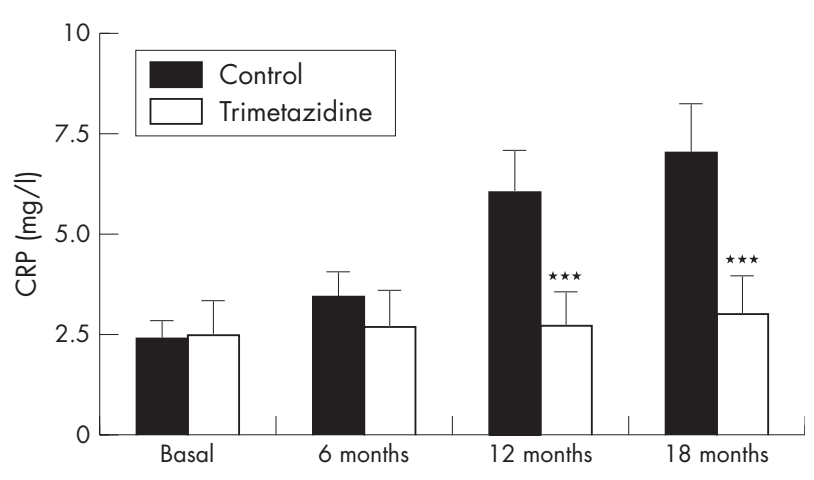

Figure 3 Changes in $\mathrm{C}$ reactive protein (CRP) plasma concentrations. Data are mean (SD). ${ }^{* * *} \mathrm{p}<0.001 v$ control.

Compared with control patients maintained with their usual treatment, trimetazidine treated patients' functional improvement was associated with a significant increase in LVEF and with a significant effect on ventricular remodelling. These effects were significant starting from 12 months and were maintained after 18 months of treatment. During the evaluation period, $\mathrm{C}$ reactive protein plasma concentrations remained stable with trimetazidine treatment and were, in contrast, significantly increased in control patients. Trimetazidine treatment was not modified or discontinued during the evaluation period. No significant adverse event related to the study treatments occurred during the study. Thus, patients with ischaemic dilated cardiomyopathy tolerated trimetazidine well during up to 18 months of treatment.

The clinical and echocardiographic data obtained in this study are in agreement with findings of Brottier et al, ${ }^{7}$ who reported improved LVEF and cardiac volumes in patients with severe ischaemic cardiomyopathy after six months' treatment with trimetazidine. Recently, Fragasso et al ${ }^{12}$ and Rosano et al ${ }^{13}$ reported relevant improvement of clinical status and LVEF in diabetic patients with ischaemic dilated cardiomyopathy after only six months of treatment with trimetazidine; the apparent discrepancy with our data, in which LVEF improved significantly after 12 months' treatment, may result from the differences in patients' characteristics and, particularly, in the low prevalence of diabetes among our patients (about 20\%).

It is suggested that the effects observed here with trimetazidine are related to the peculiar mechanism of action of the drug. Trimetazidine is one of a new group of pharmacological compounds whose myocardial anti-ischaemic effect is achieved independently from the changes in the oxygen supply to demand ratio. ${ }^{134}$ The anti-ischaemic effect of trimetazidine is obtained at the cellular level, by shifting the energy substrate reference from fatty acid oxidation to glucose oxidation.

In experimental conditions, trimetazidine exerts a cardioprotective effect by rapidly restoring the phosphorylation processes, protecting cardiac cells against intracellular acidosis, preventing the intracellular accumulation of sodium and calcium ions, and by reducing oxidative damage. ${ }^{14}{ }^{15}$ All these properties observed experimentally with trimetazidine may contribute to protecting the myocardial cell against necrosis and apoptosis. These two steps are considered fundamental in regulating LV modelling and the progressive decline of the contractile function that occurs during the normal evolution of dilated cardiomyopathy. ${ }^{16}$

The preservation of LVEF observed in trimetazidine treated patients may be explained, at least partly, by the effects of the drug in maintaining the integrity of cell membranes as well as mitochondrial structure and function. ${ }^{17}$ In addition, the increase of glucose oxidation induced by trimetazidine may in turn enhance the resynthesis of glycolytic ATP and improve contractility and microvascular function. Lastly, it is also possible that, after trimetazidine treatment, chronically hibernating cells increase their energy metabolism, become effective in producing contractile activity, and thus contribute to improving the contractile response and to limiting further decline of LV function. Data on patients with ischaemic cardiomyopathy confirmed that trimetazidine treatment improved the contractile response to a challenge of dobutamine, without inducing any haemodynamic changes. ${ }^{8}$ This amelioration of contractility is also evident during dobutamine induced ischaemic dysfunction in patients with coronary artery disease and normal LV systolic function at rest. ${ }^{18}$

In addition to these effects, we observed that $C$ reactive protein plasma concentrations remained stable throughout the study in patients receiving trimetazidine, whereas they increased significantly with time in patients receiving their standard treatment. $\mathrm{C}$ reactive protein is a biological marker commonly assessed in medical practice to evaluate the systemic inflammatory process. A proinflammatory state is recognised in chronic heart failure and the degree of immune activation corresponds to disease severity and prognosis. Peak $\mathrm{C}$ reactive protein concentration measured during myocardial infarction has been shown to be a strong predictor of global and heart failure mortality during the following year. ${ }^{19}$ In patients with heart failure, higher $\mathrm{C}$ reactive protein concentrations have also been related to higher rates of hospital readmission and mortality. ${ }^{20}$

Although our data need to be confirmed with further specific investigations, they suggest that the inflammatory process may have been limited in the patient sample treated with trimetazidine during the 18 month evaluation period. If the relevance of improvement of LV function and clinical status appears prevalent, potential anti-inflammatory effects have been previously reported with trimetazidine in experimental conditions. ${ }^{21}$ In a rabbit model of ischaemia and reperfusion, the authors reported that trimetazidine reduced neutrophil cell accumulation in the reperfused post-ischaemic myocardium. In the clinical setting, whether the effect of trimetazidine on $\mathrm{C}$ reactive protein concentrations is related to its direct action on the inflammatory process or results from its anti-ischaemic properties needs to be elucidated. Furthermore, the reduced inflammatory process may be related to the better metabolic status of either myocytic or endothelial cells; this, in turn, provides a better explanation of their physiological functions (contraction and coronary circulation, respectively), less cellular damage and, lastly, less tissue reaction and inflammation.

In conclusion, treatment with trimetazidine added to the usual treatment for up to 18 months was well tolerated and induced functional improvement in patients with ischaemic dilated cardiomyopathy. Trimetazidine treatment was associated with a significant improvement of LV function and the remodelling process. Results also suggest that the inflammatory response was limited in patients treated with trimetazidine. The effects of trimetazidine on energy metabolism, hydroionic balance, coronary microcirculation, and oxidative stress contribute positively to its beneficial effects on LV remodelling and long term cardioprotection observed in this study.

\footnotetext{
Authors' affiliations

P Di Napoli, A A Taccardi, Department of Cardiology, Intensive Care Unit, Casa di Cura Villa Pini d'Abruzzo, Chieti, Italy

A Barsotti, Department of Internal Medicine, University of Genoa, Genoa, Italy
} 


\section{REFERENCES}

1 Sellier P. Chronic effects of trimetazidine on ergometric parameters in effort angina. Cardiovasc Drug Ther 1990;4:822-3.

2 Detry JM, Sellier P, Pennaforte S, et al. Trimetazidine: a new concept in the treatment of angina. Comparison with propranolol in patients with stable angina. Br J Clin Pharmacol 1994;37:279-88.

3 Kantor PF, Lucine A, Kozak R, et al. The anti-anginal drug trimetazidine shifts cardiac energy metabolism from fatty acid oxidation to glucose oxidation by inhibiting mitochondrial long-chain 3-ketoacyl coenzyme A thiolase. Circ Res 2000;86:580-8.

4 Allibardi S, Chierchia SL, Margonato V, et al. Effects of trimetazidine on metabolic and functional recovery of postischemic rat heart. Cardiovasc Drug Ther 1998; 12:543-9.

5 De Leiris J, Boucher F. Rationale for trimetazidine administration in myocardial ischemia-reperfusion syndrome. Eur Heart $J$ 1993; 14(suppl G):34-40.

6 Guarnieri C, Muscari C. Beneficial effects of trimetazidine on mitochondrial function and superoxide production in the cardiac muscle of monocrotalinetreated rats. Biochem Pharmacol 1988;24:4685-8.

7 Brottier L, Barat L, Combe C, et al. Therapeutic value of a cardioprotective agent in patients with severe ischemic cardiomyopathy. Eur Heart $J$ 1990;1 1:207-12.

8 Belardinelli R, Purcaro A. Effects of trimetazidine on the contractile response of chronically dysfunctional myocardium to low-dose dobutamine in ischemic cardiomyopathy. Eur Heart J 2001;22:2164-70.

9 Liuzzo G, Biasucci LM, Gallimore JR, et al. The prognostic value of C-reactive protein and serum amyloid a protein in severe unstable angina. N Engl J Med 1994;331:417-24.

10 Libby P, Ridker PM, Maseri A. Inflammation and atherosclerosis. Circulation 2002; 105:1135-43.
11 Maseri A. Inflammation, atherosclerosis, and ischemic events: exploring the hidden side of the moon. N Engl J Med 1997;336:1014-6

12 Fragasso G, Piatti PM, Monti L, et al. Short and long-term beneficial effects of partial free fatty acid inhibition in diabetic patients with ischemic dilated cardiomyopathy. Am Heart J 2003;146:E1-8.

13 Rosano GM, Vitale C, Sposato B, et al. Trimetazidine improves left ventricular function in diabetic patients with coronary artery disease: a double-blind placebo-controlled study. Cardiovasc Diabetol 2003;2:16.

14 Renaud JF. Internal $\mathrm{pH}, \mathrm{Na}+$ and $\mathrm{Ca}++$ regulation by trimetazidine during cardiac cell acidosis. Cardiovasc Drug Ther 1988;1:677-86.

15 Ferrari R, Pepi P, Ferrari F, et al. Metabolic derangement in ischemic heart disease and its therapeutic control. Am J Cardiol 1998;82:2K-13K.

16 Abbate A, Bussani R, Biondi-Zoccai GG, et al. Persistent infarct-related artery occlusion is associated with an increased myocardial apoptosis at postmortem examination in humans late after an acute myocardial infarction. Circulation 2002;106:1051-4.

17 Guarnieri C, Muscari C. Effect of trimetazidine on mitochondrial function and oxidative damage during reperfusion of ischemic hypertrophied myocardium. Pharmacology 1993;46:324-31.

18 Lu C, Dabrowski P, Fracasso G, et al. Effects of trimetazidine on ischemic left ventricular dysfunction in patients with coronary artery disease: a doubleblind, placebo-controlled, crossover study. Am J Cardiol 1998;82:898-901.

19 Berton G, Cordiano R, Palmieri R, et al. C-reactive protein in acute myocardial infarction: association with heart failure. Am Heart J 2003;145:1094-101.

20 Alonso-Martinez JL, Llorente-Diez B, Echegaray-Agara M, et al. C-reactive protein as a predictor of improvement and readmission in heart failure. Eur J Heart Fail 2002;4:331-6.

21 Williams FM, Tanda K, Kus M, et al. Trimetazidine inhibits neutrophil accumulation after myocardial ischemia and reperfusion in rabbits. J Cardiovasc Pharmacol 1993;22:828-33.

\section{IMAGES IN CARDIOLOGY}

\section{Traumatic pericardial effusion caused by a safety pin}

A 27 year old man was admitted to hospital having taken an overdose of diazepam. He was managed conservatively and admitted for overnight observation and psychiatric assessment. He had a past history of substance abuse and was a registered methadone user.

The following day, unhappy at the treatment that he was receiving, the patient picked up a safety pin that was located by the side of his bed. He proceeded to straighten out the pin to a length of $9 \mathrm{~cm}$ and stab himself in the chest (upper panel). It was apparent to the medical team that the pin was moving in time with the heart beat of the patient. There was no haemodynamic compromise.

Transthoracic echocardiography showed that the pin had breached the pericardium and pierced the heart at the apex of the right ventricle, with the tip lodged in the apical segment of the interventricular septum (lower panel: LV, left ventricle; $\mathrm{RV}$, right ventricle). A $1.3 \mathrm{~cm}$ rim of pericardial fluid was also evident. The pin was successfully removed by the cardiothoracic team. Repeat echocardiography showed that the pericardial effusion had not increased in size. The patient was observed on the cardiothoracic unit for 24 hours and then transferred to the psychiatric unit.

Cardiac tamponade is often a fatal complication of penetrating mediastinal injury. In some patients, the classically described triad (muffled heart sounds, distended neck veins, and hypotension) is often absent because of concurrent hypovolaemia. The diagnostic procedure of choice is transthoracic echocardiography as chest radiography is often normal.

\section{C Shirodaria}

$\mathrm{H}$ Becher

cheerag.shirodaria@cardiov.ox.ac.uk
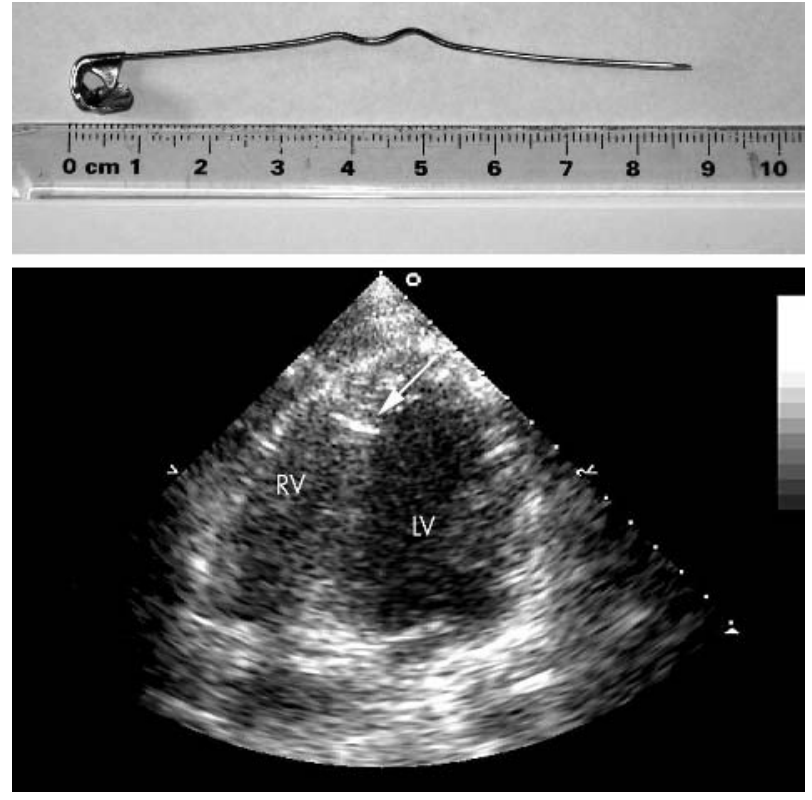